\title{
Phytoplankton trigger the production of cryptic metabolites in the marine actinobacteria Salinispora tropica.
}

\author{
Audam Chhun ${ }^{1, \#}$, Despoina Sousoni ${ }^{1}$, Maria del Mar Aguiló-Ferretjans ${ }^{2}$, Lijiang Song ${ }^{3}$, Christophe \\ Corre ${ }^{1,3, \#, ~ J o s e p h ~ A . ~ C h r i s t i e-O l e z a ~}{ }^{1,2,4, \#}$
}

\author{
${ }^{1}$ School of Life Sciences, University of Warwick, Coventry, UK \\ ${ }^{2}$ University of the Balearic Islands, Palma, Spain \\ ${ }^{3}$ Department of Chemistry, University of Warwick, Coventry, UK \\ ${ }^{4}$ IMEDEA (CSIC-UIB), Esporles, Spain \\ \#Corresponding authors: a.chhun@warwick.ac.uk, c.corre@warwick.ac.uk and joseph.christie@uib.eu
}

\begin{abstract}
Bacteria from the Actinomycete family are a remarkable source of natural products with pharmaceutical potential. The discovery of novel molecules from these organisms is, however, hindered because most of the biosynthetic gene clusters (BGCs) encoding these secondary metabolites are cryptic or silent and are referred to as orphan BGCs. While coculture has proven to be a promising approach to unlock the biosynthetic potential of many microorganisms by activating the expression of these orphan BGCs, it still remains an underexplored technique. The marine actinobacteria Salinispora tropica, for instance, produces valuable compounds such as the anti-cancer molecule salinosporamide $A$ but half of its putative BGCs are still orphan. Although previous studies have looked into using marine heterotrophs to induce orphan BGCs in Salinispora, the potential impact of co-culturing marine phototrophs with Salinispora has yet to be investigated. Following the observation of clear antimicrobial phenotype of the actinobacterium on a range of phytoplanktonic organisms, we here report the discovery of novel cryptic secondary metabolites produced by S. tropica in response to its co-culture with photosynthetic primary producers. An approach combining metabolomics and proteomics revealed that the photosynthate released by phytoplankton influences the biosynthetic capacities of $S$. tropica with both production of new molecules and the activation of orphan BGCs. Our work pioneers the use of phototrophs as a promising strategy to accelerate the discovery of novel natural products from actinobacteria.
\end{abstract}

\section{Importance}

The alarming increase of antimicrobial resistance has generated an enormous interest in the discovery of novel active compounds. The isolation of new microbes to untap novel natural products is currently hampered because most biosynthetic gene clusters (BGC) encoded by these microorganisms are not expressed under standard laboratory conditions, i.e. monocultures. Here we show that co-culturing can be an easy way for triggering silent BGC. By combining state-of-the-art metabolomics and high-throughput proteomics, we characterized the activation of cryptic metabolites and silent biosynthetic gene clusters in the marine actinobacteria Salinispora tropica by the presence of phytoplankton photosynthate. We further suggest a mechanistic understanding of the antimicrobial effect this actinobacterium has on a broad range of prokaryotic and eukaryotic phytoplankton species and reveal a promising candidate for antibiotic production. 


\section{Introduction}

Soil actinomycetes are a rich source of drug-like natural products, to which we owe up to $70 \%$ of all microbial antibiotics used today (Bérdy, 2005). Identification of novel secondary metabolites from this extensively studied family has, however, stalled over the last few decades as a result of the recurring rediscovery of already known compounds. This has led in recent years to a thriving interest for the study of new microorganisms, with the rational that ecologically distinct microorganisms produce equally distinct secondary metabolites (Molinski et al., 2009; Wilson and Brimble, 2009). For instance, the heterotrophic bacteria Salinispora drew particular attention when discovered, as it was the first obligate marine actinomycete described (Jensen et al., 1991; Mincer et al., 2002, Jensen \& Mafnas, 2006) and has since proven to be an important source of new natural products for the pharmaceutical industry (Maldonado et al., 2005; Feling et al., 2003; Buchanan et al., 2005; Asolkar et al., 2010). Despite the increasing number of novel strains identified with promising biosynthetic capacities, many hurdles in natural product discovery remain. Most of these microbial secondary metabolites are encoded by groups of colocalized genes, called biosynthetic gene clusters (BGCs), which are now more easily identified because of the improvement in sequencing technologies and bioinformatic tools (Medema et al., 2011). The majority of these discovered BGCs, however, have yet to be linked to their products and are called orphan BGCs. They are generally considered to be either silent - because of a low level of expression or inactivation of their biosynthetic genes - or the metabolites they produce are cryptic difficult to detect and isolate (Reen et al., 2015; Rutledge and Challis, 2015). The observation of numerous orphan BGCs in genome-sequenced microorganisms has resulted in a growing interest in developing biological or chemical means to activate such clusters (Abdelmohsen et al., 2015; Onaka, 2017). One of the simplest and most efficient methods described in the literature relies on co-cultivation of different microbes to elicit novel natural product biosynthesis (Slattery et al., 2001; Bertrand et al., 2014).

The genome of the marine actinomycete Salinispora tropica comprises at least 20 putative BGCs of which 11 are orphan (Table 1, Penn et al., 2009, Udwary et al., 2007). Recent studies have shown that Salinispora co-inoculated with various marine heterotrophs could produce one or several antimicrobial compounds, which remain uncharacterized as traditional analytical chemistry methods did not allow their identification and no candidate BGC was proposed (Patin et al., 2016; Patin et al., 2018). While co-culturing appears to be a promising mean to activate orphan BGCs in Salinispora, it remains an underexplored technique to unravel the biosynthetic potential of this genus. Additionally, little has been done to establish the BGCs that are activated under such culturing conditions. Combining metabolomics with proteomics analyses has proven successful in linking novel compounds to active orphan BGCs in several Streptomyces species, but has not yet been applied to the genus Salinispora (Schley et al., 2006; Gubbens et al., 2014; Owens et al., 2014).

Here we report the discovery of novel cryptic secondary metabolites produced by S. tropica CNB-440. By using an approach combining metabolomics and proteomics, we investigated how marine microbial phototrophs, and their photosynthate, induce the production of new metabolites and activate the expression of orphan BGCs in S. tropica. This strategy confirms microbial interactions as a promising and simple approach for future discovery of novel natural products. 
Material and methods

\section{Culture conditions and cell abundance monitoring}

\subsection{Strains and growth media}

Axenic marine phototrophs Synechococcus sp. WH7803, Emiliania huxleyi RCC1242 and Phaeodactylum tricornutum CCAP1055/1 were routinely grown in Artificial Seawater (ASW, Wilson et al., 1996), K-media (Probert and Houdan, 2004), and F/2 media (Guillard et al., 1975), respectively. Cultures were set-up in Falcon $25 \mathrm{~cm}^{2}$ rectangular culture flasks with vented caps containing $20 \mathrm{ml}$ of media and incubated at a constant light intensity of $10 \mu \mathrm{mol}$ photons $\mathrm{m}^{-2} \mathrm{~s}^{-1}$, at $22{ }^{\circ} \mathrm{C}$ with orbital shaking (140 rpm). Salinispora tropica CNB-440 was grown in marine broth (MB, Difco), and incubated at $30^{\circ} \mathrm{C}$ with orbital shaking (220 rpm). The S. tropica mutants $s a l A^{-}$and $s a l L^{-}$were generously provided by the Moore Laboratory, USA (Eustáquio et al., 2008; Eustáquio et al., 2009).

\subsection{Co-culture setup}

Salinispora cells were grown to late exponential phase in $10 \mathrm{ml}$ of MB before washing them three times with sterile mineral media, as appropriate for each phototroph, and finally resuspending the washed cell pellet in $10 \mathrm{ml}$ of mineral media. Exponentially growing axenic phototroph cells and the washed Salinispora were co-inoculated in fresh media to a concentration of $10 \%(\mathrm{v} / \mathrm{v})$ and $20 \%(\mathrm{v} / \mathrm{v})$, respectively. Salinispora cells were also washed and resuspended in a conditioned Synechococcus supernatant (SUPSYN), when required for the metabolomic and proteomic analyses. To obtain the conditioned supernatant, Synechococcus cultures were incubated for 35 days as described above before centrifugation ( $4000 \times g$ for $10 \mathrm{~min}$ at room temperature) and further filtration through $0.22 \mu \mathrm{m}$ pore size filters to remove cells and particulate organic matter. Washed Salinispora cells were used to inoculate SUPSYN and $M B$, and cultures were incubated at $22^{\circ} \mathrm{C}$ with shaking $(140 \mathrm{rpm})$ and a light intensity of $10 \mu \mathrm{mol}$ photons $\mathrm{m}^{-2} \mathrm{~s}^{-1}$. For the physically separated Synechococcus-Salinispora co-cultures using the porous filters, cells were grown in $24 \mathrm{~mm}$ transwell with $0.4 \mu \mathrm{m}$ pore polycarbonate membrane inserts (Corning). Synechococcus cells were inoculated in the well to a concentration of $20 \%(\mathrm{v} / \mathrm{v})$ and Salinispora in the insert to a concentration of $55 \%(\mathrm{v} / \mathrm{v})$.

\subsection{Flow cytometry}

Phototroph cell abundance was monitored using their autofluorescence by flow cytometry using a LSR Fortessa Flow Cytometer (BD) instrument, and the BD FACSDiva acquisition software (BD). Cells were detected and gated using ex. $488 \mathrm{~nm}-\mathrm{em} .710 / 50 \mathrm{~nm}$ at voltage $370 \mathrm{~V}$, and ex. $640 \mathrm{~nm}-$ em. filter $670 / 14 \mathrm{~nm}$ at voltage $480 \mathrm{~V}$. To remove any Salinispora cell aggregates that would block the flow cell, samples were pre-filtered through a sterile mesh with pore size of $35 \mu \mathrm{m}$ (Corning) prior to analysis.

\section{Metabolomic analysis}

\subsection{Sample preparation}

The culture supernatants were analyzed by non-targeted metabolomic using either raw or concentrated supernatants. Raw supernatants were collected by sampling $200 \mu \mathrm{l}$ of $0.22 \mu \mathrm{m}$ filtered culture milieu, prior to being mixed with an equal volume of HPLC-grade methanol. For concentrating the supernatant, cells from 10 to $100 \mathrm{~mL}$ of cultures were removed by centrifugation (4,000 $\mathrm{x} g$ for $15 \mathrm{~min}$ ) followed by a filtering step using $0.22 \mu \mathrm{m}$ vacuum filter bottle system (Corning). Pre-purification of the compounds of interest from the supernatants was carried out by solid phase extraction using C18-silica. Using a 90:10 A/B mobile phase 
(where $A$ is water with $0.1 \%$ formic acid and $B$ is methanol with $0.1 \%$ formic acid) the undesired polar molecules and salts passed through the silica while the compounds of interest were retained and later collected following elution with a 10:90 A/B mobile phase. The obtained fractions were dried under reduced pressure at $40{ }^{\circ} \mathrm{C}$ (in a speed-vac) and resuspended in 1-3 $\mathrm{mL}$ of 50:50 HPLC-grade methanol/water solution. All samples were stored in snap-seal amber glass vials (Thames Restek) and kept at $-20^{\circ} \mathrm{C}$ until analysis.

\subsection{Low-resolution LC-MS}

Metabolites present in the cultures were routinely analyzed by reversed-phase liquid chromatography. A Dionex UltiMate 3000 HPLC (ThermoScientific) coupled with an amaZon SL Ion Trap MS (Bruker) was used. A Zorbax Eclipse Plus C18 column with dimensions $4.6 \mathrm{~mm}$ $x 150 \mathrm{~mm}, 5 \mu \mathrm{m}$ particle size (Agilent Technologies) was employed for metabolite separation with a linear gradient of 95:5 A/B to 30:70 A/B over 5 minutes, followed by second linear gradient to $20: 80 \mathrm{~A} / \mathrm{B}$ over 10 minutes with a flow rate of $1 \mathrm{ml} \mathrm{min}^{-1}$ (Mobile phase $A$ : water with $0.1 \%$ formic acid, B: methanol with $0.1 \%$ formic acid). The mass spectrometer was operated in positive ion mode with a $100-1000 \mathrm{~m} / \mathrm{z}$ scan range. The injected volume was 10 $\mu \mathrm{L}$ at a temperature of $25{ }^{\circ} \mathrm{C}$. Data was processed with the Bruker Compass DataAnalysis software version 4.2 (Bruker).

\subsection{High-resolution LC-MS}

To acquire molecular formulae information, samples were analyzed using an Ultra-high resolution MaXis II Q-TOF mass spectrometer equipped with electrospray source coupled with Dionex 3000RS UHPLC was employed (Bruker). A reverse phase C18 column (Agilent Zorbax, 100x2.1 mm, $1.8 \mu \mathrm{m}$ ) and a guard column (Agilent C18, 10x2.1 mm, $1.8 \mu \mathrm{m}$ ) were used for separation applying a linear gradient of 95:5 A/B to 0:100 A/B over 20 minutes (Mobile phase $A$ : water with $0.1 \%$ formic acid, B: acetonitrile with $0.1 \%$ formic acid). The injected volume was $2 \mu \mathrm{L}$, and the flow rate was $0.2 \mathrm{ml} \mathrm{min}^{-1}$. At the beginning of each run, $7.5 \mu \mathrm{l}$ of $10 \mathrm{mM}$ of sodium formate solution was injected for internal calibration. The mass spectrometer was operated in positive ion mode with a $50-2500 \mathrm{~m} / \mathrm{z}$ scan range. MS/MS data was acquired for the three most intense peaks in each scan.

\section{Proteomic analysis}

\subsection{Preparation of cellular proteome samples}

Cultures were set up as described above and incubated for 5 days after which cells were collected by centrifuging $10 \mathrm{~mL}$ of culture at $4,000 \times g$ for $10 \mathrm{~min}$ at $4{ }^{\circ} \mathrm{C}$. Cell pellets were placed on dry ice before storing at $-20{ }^{\circ} \mathrm{C}$ until further processing. The cell pellets were resuspended in $200 \mu \mathrm{L} 1 \times$ NuPAGE lithium dodecyl sulfate (LDS) sample buffer (ThermoFischer Scientific), supplemented with $1 \%$ ß-mercaptoethanol. Cell pellets were lysed by bead beating ( $2 \times 45 \mathrm{sec}$ and $1 \times 30 \mathrm{sec}$ at $6.0 \mathrm{~m} / \mathrm{s}$ ) and sonication ( $5 \mathrm{~min}$ ), followed by three successive 5 -min incubations at $95{ }^{\circ} \mathrm{C}$ with short vortex steps in between. Cell lysates containing all proteins were loaded on an SDS-PAGE precast Tris-Bis NuPAGE gel (Invitrogen), using MOPS solution (Invitrogen) as the running buffer. Protein migration in the SDS-PAGE gel was performed for 5 min at $200 \mathrm{~V}$, to allow removal of contaminants and purification of the polypeptides. The resulting gel was stained using SimplyBlue SafeStain (Invitrogen) to visualize the cellular proteome. The gel bands containing the cellular proteome were excised and stored at $-20^{\circ} \mathrm{C}$ until further processing. 

performed using dithiothreitol and iodoacetamide, respectively, after which proteins were in-

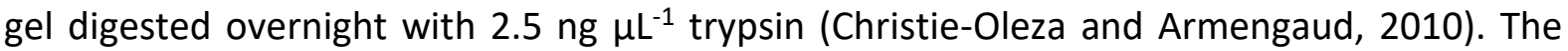
resulting peptide mixture was extracted by sonication of the gel slices in a solution of $5 \%$ formic acid in $25 \%$ acetonitrile, and finally concentrated at $40{ }^{\circ} \mathrm{C}$ in a speed-vac. For mass spectrometry analysis, peptides were resuspended in a solution of $0.05 \%$ trifluoroacetic acid in $2.5 \%$ acetonitrile prior to filtering using a $0.22 \mu \mathrm{m}$ cellulose acetate spin column. Samples were analyzed by nanoLC-ESI-MS/MS with an Ultimate 3000 LC system (Dionex-LC Packings) coupled to an Orbitrap Fusion mass spectrometer (Thermo Scientific) using a 60 min LC separation on a $25 \mathrm{~cm}$ column and settings as previously specified (Christie-Oleza et al., 2015).

\subsection{Proteomic data analysis}

Raw mass spectral files were processed for protein identification and quantification using the software MaxQuant (version 1.5.5.1; Cox and Mann, 2008) and the UniProt database of $S$. tropica CNB-440 (UP000000235). Quantification and normalization of spectral counts was done using the Label-Free Quantification (LFQ) method (Cox et al., 2014). Samples were matched between runs for peptide identification and other parameters were set by default. Data processing was completed using the software Perseus (version 1.5.5.3). Proteins were filtered by removing decoy and contaminants and were considered valid when present in at least two replicates for one condition. The relative abundance of each protein was calculated using protein intensities transformed to a logarithmic scale with base 2 and normalized to protein size. Variations in protein expression were assessed with a two-sample T-test, with a false discovery rate (FDR) $q$ below 0.05 and a $\log (2)$ fold change above 2 (Supplementary File S1).

\section{Results}

\section{Salinispora tropica has antimicrobial activity on a diverse range of marine phototrophs}

Unlike other heterotrophs, which usually enhance the growth of phototrophic organisms when in co-culture (e.g. Christie-Oleza et al., 2017; Sher et al., 2011), S. tropica showed a clear antimicrobial activity on marine phytoplankton (Fig. 1, A). All three phototrophic model species tested, namely the cyanobacteria Synechococcus sp. WH7803, the coccolithophore Emiliana huxleyi and the diatom Phaeodactylum tricornutum, showed a strong decline in the presence of $S$. tropica, being especially remarkable for the two former species (Fig. 1, A). While also affected, the diatom $P$. tricornutum was not killed by $S$. tropica but, instead, its cells densities were significantly maintained one order of magnitude lower than when incubated axenically.

We were therefore interested in characterizing the nature of this inhibition. While other Salinispora species, such as Salinispora arenicola, are known to biosynthesize antibiotic molecules (Asolkar et al., 2010), no antimicrobial compound has yet been characterized in $S$. tropica CNB-440. Previous studies have shown, however, that $S$. tropica is able to outcompete other heterotrophs in co-culture by secreting siderophores leading to iron depletion (Patin et al., 2016). To evaluate whether iron sequestration could explain the negative interactions observed in the present phototroph-Salinispora system, we supplemented the co-cultures with increasing concentrations of iron (Supplementary Fig. S1). The results obtained suggest 
that the antimicrobial phenotype was not due to siderophore activity, as saturating amount of iron could not rescue the growth of the phototrophs.

We then hypothesized that a yet unknown antimicrobial compound, to which our photosynthetic microorganisms are sensitive to, could be produced by $S$. tropica. To test this assumption, we setup co-cultures in which S. tropica and Synechococcus were physically separated by a porous filter, preventing direct cell-to-cell interactions while allowing the diffusion of small molecules (Fig. 1, B). S. tropica was still able to impair Synechococcus proliferation in these experimental conditions, confirming that a secreted molecule was causing the death of the phototroph.

\section{Phototrophs elicit the production of novel cryptic metabolites in S. tropica}

We analyzed the co-culture supernatants using non-targeted metabolomics to identify the pool of secondary metabolites secreted by S. tropica in response to the different phototrophs. The Synechococcus-S. tropica co-culture revealed eight molecular ions that were not present in the respective axenic cultures (Fig. 2, A). These molecules were further characterized by high-resolution MS/MS analysis, from which we generated empirical chemical formulae, allowing us to assign most of them to two subgroups of related compounds being: (i) ions $\mathbf{1}$, 2, 5 and 8; and (ii) ions 4, 6 and 7 (Fig. 2, A and Supplementary Table S1).

Ions 1, 2, 5 and 8 were derivatives of salinosporamide; a well-characterized molecule produced by $S$. tropica that presents a unique fused $\gamma$-lactam- $\beta$-lactone bicyclic ring structure (Feling et al., 2003), and that is now being tested as a drug because of its anti-cancer properties. Molecules $\mathbf{5}$ and $\mathbf{8}$ are consistent with known degradation products of salinosporamide A and B, respectively (Denora et al., 2007; Supplementary Fig. S2), while molecules $\mathbf{1}$ and $\mathbf{2}$ are proposed to result from the nucleophilic addition of Tris (the buffering agent used in the ASW culture medium) to the lactone ring of salinosporamide $A$ and $B$, respectively (Supplementary Fig. S2). These salinosporamide sub-products were further confirmed by their absence when i) Tris was not added (Supplementary Fig. S3), or ii) salinosporamide mutants that no longer produced these metabolites, i.e. salA $\mathrm{A}^{-}$and salL' $^{-}$ (Eustáquio et al., 2009), were used (Supplementary Fig. S4). In order to test the activity of salinosporamide and its derivatives on the phototrophs, we co-cultured Synechococcus with both salinosporamide mutants. Salinosporamide and its derivatives were not responsible for the antimicrobial activity as both deficient mutants were still able to inhibit the phototroph (Supplementary Fig. S5).

The second group of ions, i.e. peaks 4, 6 and 7, were also related. Molecule 6 gave a $m / z$ value of $435.2609[\mathrm{M}+\mathrm{H}]^{+}$; based on the accuracy of this value and the isotopic pattern the empirical chemical formula $\mathrm{C}_{22} \mathrm{H}_{35} \mathrm{~N}_{4} \mathrm{O}_{5}$ was predicted by the DataAnalysis software (Table 2). The predicted formula for molecule 4 suggests that, with a 28.9900 Da mass difference when compared to 6 , the compound had lost one hydrogen and gained an atom of nitrogen and oxygen. MS/MS analyses confirmed that both molecules 4 and 6 had an identical molecular fragment (i.e. $\mathrm{m} / \mathrm{z} 276.1600 \pm 0.0001[\mathrm{M}+\mathrm{H}]^{+}$, with the empirical chemical formula $\mathrm{C}_{16} \mathrm{H}_{22} \mathrm{NO}_{3}$ ), indicating that the two molecules share a core backbone (Table 2). Similarly, molecule 7 had the same chemical formula than 6 but with the addition of a methyl group (14.0155 Da mass difference; Table 2). Molecule 3 did not share an obvious link to any other metabolites and, therefore, was considered a new biosynthesized product of Salinispora 
(Table 2). Most interestingly, the search for compounds with the same molecular formulae as 3, 4, $\mathbf{6}$ or $\mathbf{7}$ in multiple databases (e.g. Reaxys, SciFinder, Dictionary of NP) returned no known natural product, suggesting that they are novel compounds. Unfortunately, despite multiple attempts, the isolation of these molecules has so far proven too challenging for their structural elucidation.

The production of these novel compounds was only triggered by the presence of the phototrophs as they were only detected in the co-cultures of all three phototrophs (Fig. 2, A and $\mathbf{B})$, but not when grown in mono culture - as shown by the absence of these metabolites when S. tropica was grown alone in mineral ASW or nutrient rich media MB (Fig. 2, C and D). Furthermore, we confirmed that the supernatant of a phototroph culture - containing the photosynthate - was enough to induce such metabolite production (Fig. 2, D).

\section{Photosynthate triggers the expression of orphan gene clusters in S. tropica}

Having detected novel secondary metabolites produced by $S$. tropica in response to phototroph-released photosynthate, we set out to investigate how it affected the induction of its BGCs. To this end, we analyzed and compared the proteome of S. tropica when grown in presence of the phytoplankton's photosynthate - i.e. in conditioned Synechococcus supernatant - and in nutrient rich broth - i.e. marine broth. Surprisingly, we were able to detect proteins encoded by almost all of S. tropica's BGCs, including 10 of its 11 orphans BGCS (Fig. 3, A).

Of particular interest were the orphan BGCs pks3 and nrps1, for which we detected $72 \%$ $(18 / 25)$ and $42 \%(14 / 33)$ of their encoded proteins, respectively (Fig. 3, A). Moreover, the pks3 BGC was noticeably highly detected as eight of its detected proteins showed a relative abundance above $0.1 \%$ (Supplementary Table S2). While it has been previously suggested that $p k s 3$ may produce a spore pigment polyketide, very little experimental evidence is available in the literature, and the product of $p k s 3$ had not been confirmed (Kersten et al., 2013). On the other hand, the non-ribosomal peptide synthetase (NRPS) gene cluster nrps1 has only been predicted to produce a non-ribosomal dipeptide (Penn et al., 2009). Intriguingly, the most abundant proteins detected from this nrps1 BGC were the nonribosomal peptide synthetase $(\mathrm{A} 4 \mathrm{X} 2 \mathrm{Q} 0)$, the condensation domain-containing protein (A4X2R5) and an ATP-dependent Clp protease subunit (A4X2S2), with a relative abundance of $0.004 \%, 0.001 \%$ and $0.121 \%$, respectively (Table 3 ). While the two former are thought to direct the biosynthesis of the non-ribosomal peptide, the later may be involved in conferring resistance to the synthesized antimicrobial compound (Kirstein et al., 2009), as further discussed below.

The already characterized lom and sal BGCs were also abundantly detected with $81 \%(47 / 58)$ and $77 \%(23 / 30)$ of their encoded proteins detected, respectively, some representing high relative abundances within the proteome (Fig. 3, A). The BGC lom is linked to the cytotoxic glycoside lomaiviticin molecule (Kersten et al., 2013). However, this metabolite previously showed no antimicrobial activity on co-cultured heterotrophic organisms (Patin et al., 2018) and, hence, it is unlikely to cause the antimicrobial phenotype observed on the phototrophs in this study. The high abundance of the sal cluster, producing the salinosporamide compound, is not surprising given the high detection of this metabolite by LC-MS (Fig. 2, A). 
Interestingly, the comparative proteomic analysis of S. tropica grown in photosynthate versus marine broth confirmed that the detection of several BGCs rose in response to phototrophreleased nutrients, being lom and $p k s 3$ the most remarkable ones (Fig. 3, B-C). For instance, the lom cluster had $77 \%$ (36/47) of its detected proteins overexpressed in the presence of the photosynthate (Fig. 3, B). The orphan pks3 BGC was also triggered by the photosynthate, as the pivotal biosynthetic enzymes for polyketide biosynthesis, i.e. acyl-CoA ligase (A4X7T8), 3ketoacyl-ACP synthase (A4X7U0) and long-chain fatty acid-CoA ligase (A4X7U3), were upregulated (3.1, 2.6 and 4.1-fold change, respectively; Fig. 3, C and Supplementary Table S2).

\section{Discussion}

We show that S. tropica is able to inhibit the growth of both marine cyanobacteria and eukaryotic phototrophs by some, yet, unidentified mechanism (Fig. 1). This observation broadens the potential role and impact that the Salinispora genus has on marine microbial communities. Salinispora is a widely-distributed bacterium found in all tropical and subtropical oceans (Mincer et al., 2002; Bauermeister et al., 2018). While mostly inhabiting marine sediments, bacteria from this genus were also isolated from marine sponges where it is suggested they influence the sponge microbiota through the production of acyl homoserine lactone molecules and antibiotics (Singh et al., 2014; Bose et al., 2017). Similarly, different species of Salinispora were shown to possess distinct mechanisms to outcompete cooccurring marine heterotrophs in sediments, i.e. through the production of siderophores to deplete iron or antimicrobial molecules (Patin et al., 2017; Tuttle et al., 2019), although no antimicrobial compound has yet been identified for S. tropica (Patin et al., 2018). We herein provide the first evidence that Salinispora might not only directly influence heterotrophic communities, but also kill both prokaryotic and eukaryotic phytoplankton to which they are exposed, e.g. when these sediment out of the water column or phototrophs able to grow in sunlit coastal sediments.

While we were successful in identifying and obtaining the molecular formulae of novel cryptic metabolites produced in response to phytoplanktonic photosynthate (Fig. 2, Table 2), we were unable to isolate and identify the compound responsible for the antimicrobial effect on the marine phototrophs by using traditional bioactivity-guided assays with HPLC fractionation of crude extracts (data not shown). This mechanism proved similarly elusive in previous studies, where S. tropica showed an antimicrobial activity on marine heterotrophs, but the molecule responsible was not identified (Patin et al., 2016; Patin et al., 2018). The parallelism between our observations and those described in the literature suggests that the active compound(s) may be the same. We reason that the compound's instability, and/or synergic effect of several molecules required for activity, could explain the difficulty in identifying the antimicrobial agent. For instance, the large number of structurally-related metabolites resulting from the chemical reaction of salinosporamide with various compounds (i.e. water, Tris) may support this hypothesis, as the antimicrobial molecule may be similarly unstable. The diversity of products arising from a single BGC may also be due to the promiscuity of the biosynthetic enzymes utilizing structurally related primary precursors. This results in a range of compounds, each produced at lower titers than a single natural product, and ultimately hamper the isolation of sufficient amounts of the compounds of interest. Whichever the case, we show that Salinispora can produce a broad-range antibiotic able to affect both unicellular prokaryotes and eukaryotes alike, such as the marine diatom and coccolithophore tested in our study. Such a broad-range antimicrobial could suggest a mode of action affecting a 
common target present in both types of cells such as the proteasome, a proteolytic complex present in the three domains of life (Becker and Darwin, 2016).

Exploring the proteome of S. tropica exposed to photosynthate, we detected proteins encoded by almost all its BGCs, including most of its orphan BGCs (Fig. 3). Notably, the sal $B G C$, producing the salinosporamide compound, was one of the most highly expressed BGC as most of its proteins were detected with high relative abundance. This finding is in agreement with previous studies that have shown by transcriptomics that the BGC sal is highly and constitutively expressed when grown in nutrient rich $A 1$ medium (Amos et al., 2017). Also, the high expression of this BGC correlated with a noticeable detection of salinosporamide derivatives by LC-MS. The agreement between the metabolomic and proteomic data suggests that it is possible to correlate activated BGCs with the actual biosynthesis of their corresponding natural product. Therefore, the abundant detection of several orphans BGC proteins, including those from pks3 and nrps1 BGCs, may be promising candidates responsible for the biosynthesis of the cryptic metabolites detected by LC-MS and, potentially, the antimicrobial activity observed on co-cultured phototrophs.

The proteins detected from the BGC nrps1 are essential enzymes involved in non-ribosomal peptide biosynthesis, i.e. A4X2Q0, a non-ribosomal peptide synthetase (NRPS) made of a CA-PCP domain, and A4X2RS, a condensation domain-containing protein made of C-PCP-TE domain. The detection of these proteins therefore strongly suggests the actual synthesis of the non-ribosomal peptide and could well be the novel metabolites detected by LC-MS, which include four nitrogen atoms in their predicted molecular formulae (Table 2). Interestingly, the substrate specificity of A4X2Q0's A-domain is alanine and another three A-domains are encoded in the nrps1 BGC. Further work is required to elucidate the structure of this series of cryptic metabolites. From this same BGC we also detected a highly abundant ATP-dependent Clp protease proteolytic subunit (ClpP, A4X2S2) that may be providing Salinispora with selfresistance against the nrps 1 peptides. Virtually all organisms across the tree of life have a system for targeted proteolysis for protein turnover, with most bacteria, mitochondria and chloroplasts relying on a ClpP-type proteasome while eukaryotes, archaea and some actinobacteria typically possess the homologous $20 \mathrm{~S}$ proteasome structure (Becker and Darwin, 2016; Snoberger et al., 2017). The ClpP proteasome is known to be the target for certain antibiotics, including the novel acyldepsipeptide (ADEP) class (Kirstein et al., 2009), and it is common to find alternative ClpP proteasomes encoded nearby the antibioticproducing BGC to confer resistance to the host cell (Thomy et al., 2019). In a similar fashion, salinosporamide A is a $20 \mathrm{~S}$ proteasome inhibitor, to which Salinispora is resistant because of an extra copy of the proteasome beta subunit gene within the salinosporamide-producing cluster (Kale et al., 2011). We can thus reasonably infer from the presence of $c / p P$ in the nrps1 BGC that it is likely to produce an antibiotic targeting the ClpP proteasome, a class of antimicrobial compounds that has recently gained considerable attention as an attractive option to tackle multidrug resistant pathogens (Fig. 4; Momose and Kawada, 2016; Culp and Wright, 2017; Moreno-Cinos et al., 2019). We here provide the first proteomic evidence that S. tropica's nrps1 is active and may produce a promising antimicrobial compound acting as a ClpP proteasome inhibitor. The synthesis of such antibiotic would explain the antimicrobial effect of Salinispora on all marine phototrophs tested in our study as they all rely on the ClpP proteolytic machinery (Andersson et al., 2009; Jones et al., 2013; Zhao et al., 2018). Additional evidence, such as genetic inactivation of the nrps1 BGC, will confirm this mechanism. 
We show that the photosynthate released by primary producers influences the biosynthetic capacities of Salinispora, activating the expression of several orphan BGCs and inducing the production of novel metabolites. Our metabolomics analysis further confirmed the potential of co-culturing for natural product discovery as we identified novel cryptic secondary metabolites, although future work is required to elucidate the structure of the new molecules. Finally, our study extends the pool of known compounds produced by the genus Salinispora and pioneers the use of phototrophs as a promising strategy to trigger novel natural products from marine actinobacteria. We also provide a valuable insight into the biosynthetic potential of $S$. tropica with our proteomic dataset, which reveals the nrps1 BGC as a promising candidate for antibiotic production.

\section{Conflicts of interest}

The authors declare that they have no conflicts of interest.

\section{Acknowledgments} throughout the project. We also acknowledge technical support from Cleidiane Zampronio of the WPH Proteomic Facility at the University of Warwick. In addition, we thank the BBSRC/EPSRC Synthetic Biology Research Centre WISB BB/M017982/1 for access to the flow cytometer and Yin Chen for access to the LC-MS.

A.C. was supported by an MIBTP PhD scholarship (BB/M01116X/1) and D.S. by a NERC CENTA DTP studentship (NE/L002493/1). J.A.C.-O was funded by a NERC Independent Research Fellowship NE/K009044/1 and Ramón y Cajal contract RYC-2017-22452 (funded by the Ministry of Science, Innovation and Universities, the National Agency of Research, and the European Social Fund). C.C. thanks BBSRC (grant BB/M022765/1) and European Union's Horizon 2020 research No. 765147 for support. L.S. would like to acknowledge BBSRC (BB/M017982/1 and BB/R000689/1) and EPSRC (EP/P0305721/1) for financial support.

452

453

454

455

\section{References}

Abdelmohsen UR, Grkoviv T, Balasubramanian S, Kamel MS, Quinn RJ, Hentschel U: Elicitation of secondary metabolism in actinomycetes. Biotechnol Adv (2015), 33(6 Pt 1):798-811. doi: 10.1016/j.biotechadv.2015.06.003.

Andersson FI, Tryggvesson A, Sharon M, Diemand AV, Classen M, Best C, Schmidt R, Schelin J, Stanne TM, Bukau B, Robinson CV, Witt S, Mogk A, Clarke AK: Structure and function of a novel type of ATP-dependent Clp protease. J Biol Chem (2009), 284(20):13519-32. doi: 10.1074/jbc.M809588200.

Asolkar RN, Kirkland TN, Jensen PR, Fenical W: Arenimycin, an antibiotic effective again rifampin- and methicillin-resistant Staphylococcus aureus from the marine actinomycete 468 
Bauermeister A, Velasco-Alzate K, Dias T, Macedo H, Ferreira EG, Jimenez PC, Lotufo TMC, Lopes NP, Gaudêncio SP, Costa-Lotufo LV: Metabolomic fingerprinting of Salinispora from Atlantic oceanic islands. Front Microbiol (2018), 9:3021. doi: 10.3389/fmicb.2018.03021.

Becker SH, Darwin KH: Bacterial proteasomes: mechanistic and functional insights. Microbiol Mol Biol Rev (2016), 81(1). doi: 10.1128/MMBR.00036-16.

Bérdy J: Bioactive microbial metabolites. J Antibiot (2005), 58(1):1-26. doi: 10.1038/ja.2005.1.

Bertrand S, Bohni N, Schnee S, Schumpp O, Gindro K, Wolfender JL: Metabolite induction via microorganism co-culture: a potential way to enhance chemical diversity for drug discovery. Biotechnol Adv (2014), 32(6):1180-204. doi: 10.1016/j.biotechadv.2014.03.001.

Bose U, Ortori CA, Sarmad S, Barrett DA, Hewavitharana AK, Hodson MP, Fuerst JA, Shaw PN, Boden R: Production of $\mathrm{N}$-acyl homoserine lactones by the sponge-associated marine actinobacteria Salinispora arenicola and Salinispora pacifica. FEMS Microbiol Lett (2017), 364(2). doi: 10.1093/femsle/fnx002.

Buchanan GO, Williams PG, Feling RH, Kauffman CA, Jensen PR, Fenical W: Sporolides A and B: structurally unprecedented halogenated macrolides from the marine actinomycete Salinispora tropica. Org Lett (2005), 7(13):2731-4. doi: 10.1021/ol050901i.

Christie-Oleza JA, Armengaud J: In-depth analysis of exoproteomes from marine bacteria by shotgun liquid chromatography-tandem mass spectrometry: the Ruegeria pomeroyi DSS-3 case-study. Mar Drugs (2010), 8(8):2223-39. doi: 10.3390/md8082223.

Christie-Oleza JA, Scanlan DJ, Armengaud J: "You produce while I clean up", a strategy revealed by exoproteomics during Synechococcus-Roseobacter interactions. Proteomics (2015), 15(20):3454-62. doi: 10.1002/pmic.201400562.

Christie-Oleza JA, Sousoni D, Lloyd M, Armengaud J, Scanlan DJ: Nutrient recycling facilitates long-term stability of marine microbial phototroph-heterotroph interactions. Nat Microbiol (2017), 2:17100. doi: 10.1038/nmicrobiol.2017.100.

Cox J, Hein MY, Luber CA, Paron I, Nagaraj N, Mann M: Accurate proteome-wide label-free quantification by delayed normalization and maximal peptide ratio extraction, termed MaxLFQ. Mol Cell Proteomics (2014), 13(9):2513-26. doi: 10.1074/mcp.M113.031591.

Cox J, Mann M: MaxQuant enables high peptide identification rates, individualized p.p.b.range mass accuracies and proteome-wide protein quantification. Nat Biotechnol (2008), 26(12):1367-72. doi: 10.1038/nbt.1511.

Culp E, Wright GD: Bacterial proteases, untapped antimicrobial drug targets. J Antibiot (Tokyo) (2017), 70(4):366-377. doi: 10.1038/ja.2016.138. 
Denora N, Potts BC, Stella VJ: A mechanistic and kinetic study of the beta-lactone hydrolysis of Salinosporamide A (NPI-0052), a novel proteasome inhibitor. J Pharm Sci (2007), 96(8):2037-47. doi: 10.1002/jps.20835.

Dineshkumar K, Aparna V, Madhuri KZ, Hopper W: Biological activity of sporolides A and B from Salinispora tropica: in silico target prediction using ligand-based pharmacophore mapping and in vitro activity validation on HIV-1 reverse transcriptase. Chem Biol Drug Des (2014), 83(3):350-61. doi: 10.1111/cbdd.12252.

Eustáquio AS, McGlinchey RP, Liu Y, Hazzard C, Beer LL, Florova G, Alhamadsheh MM, Lechner $A$, Kale AJ, Kobayashi $Y$, Reynolds KA, Moore BS: Biosynthesis of the salinosporamide $A$ polyketide synthase substrate chloroethylmalonyl-coenzyme $A$ from S-adenosyl-Lmethionine. Proc Natl Acad Sci USA (2009), 106(30):12295-300. doi: 10.1073/pnas.0901237106.

Eustáquio AS, Pojer F, Noel JP, Moore BS: Discovery and characterization of a marine bacterial SAM-dependent chlorinase. Nat Chem Biol (2008), 4(1):69-74. doi: 10.1038/nchembio.2007.56.

Feling RH, Buchanan GO, Mincer TJ, Kauffman CA, Jensen PR, Fenical W: Salinosporamide A: a highly cytotoxic proteasome inhibitor from a novel microbial source, a marine bacterium of the new genus Salinospora. Angew Chem Int Ed Engl (2003), 42(3):355-7. doi: 10.1002/anie.200390115.

Guillard RRL: Culture of phytoplankton for feeding marine invertebrates. In: Smith W.L., Chanley M.H. (eds) Culture of Marine Invertebrate Animals. Springer, Boston, MA (1975), 2960. doi: https://doi.org/10.1007/978-1-4615-8714-9_3.

Gubbens J, Zhu H, Girard G, Song L, Florea BI, Aston P, Ichinose K, Filippov DV, Choi YH, Overkleeft HS, Challis GL, van Wezel GP: Natural product proteomining, a quantitative proteomics platform, allows rapid discovery of biosynthetic gene clusters for different classes of natural products. Chem Biol (2014). 21(6):707-18. doi: 10.1016/j.chembiol.2014.03.011.

Jensen PR, Dwight R, Fenical W: Distribution of actinomycetes in near-shore tropical marine sediments. Appl Environ Microbiol (1991), 57(4):1102-8

Jensen PR, Mafnas C: Biogeography of the marine actinomycete Salinispora. Environ Microbiol (2006), 8(11):1881-8. doi: 10.1111/j.1462-2920.2006.01093.x.

Jones BM, Iglesias-Rodriguez MD, Skipp PJ, Edwards RJ, Greaves MJ, Young JR, Elderfield H, $\mathrm{O}$ Connor CD: Responses of the Emiliania huxleyi proteome to ocean acidification. PLOS One (2013), 8(4). doi: 10.1371/journal.pone.0061868.

Kale AJ, McGlinchey RP, Lechner A, Moore BS: Bacterial self-resistance to the natural proteasome inhibitor salinosporamide A. ACS Chem Biol (2011), 6(11):1257-64. doi: $10.1021 / c b 2002544$. 
562

563

564

565

566

567

568

569

570

571

572

573

574

575

576

577

578

579

580

581

582

583

584

585

586

587

588

589

590

591

592

593

594

595

596

597

598

599

600

601

602

603

604

605

606

607

Kersten RD, Lane AL, Nett M, Richter TKS, Duggan BM, Dorrestein PC, Moore BS: Bioactivityguided genome mining reveals the lomaiviticin biosynthetic gene cluster in Salinispora tropica. ChemBioChem (2013). 14:955-962. doi: 10.1002/cbic.201300147.

Kirstein J, Hoffmann A, Lilie H, Schmidt R, Rübsamen-Waigmann H, Brötz-Oesterhelt $H$, Mogk $A$, Turgay $K$ : The antibiotic ADEP reprogrammes ClpP, switching it from a regulated to an uncontrolled protease. EMBO Mol Med (2009), 1(1):37-49. doi: 10.1002/emmm.200900002.

Maldonado LA, Fenical W, Jensen PR, Kauffman CA, Mincer TJ, Ward AC, Bull AT, Goodfellow M: Salinispora arenicola gen. nov., sp. nov. and Salinispora tropica sp. nov., obligate marine actinomycetes belonging to the family Micromonosporaceae. Int J Syst Evol Microbiol (2005), 55(Pt 5):1759-66. doi: 10.1099/ijs.0.63625-0.

Medema MH, Blin K, Cimermancic P, de Jager V, Zakrzewski P, Fischbach MA, Weber T, Takano $\mathrm{E}$, Breitling R: antiSMASH: rapid identification, annotation and analysis of secondary metabolite biosynthesis gene clusters in bacterial and fungal genome sequences. Nucleic Acids Res (2011). 39:W339-46. doi: 10.1093/nar/gkr466.

Miyanaga A, Janso JE, McDonarld L, He M, Liu H, Barbieri L, Eustáquio AS, Fielding EN, Carter GT, Jensen PR, Feng $X$, Leighton $M$, Koehn FE, Moore BS: Discovery and Assembly Line Biosynthesis of the Lymphostin Pyrroloquinoline Alkaloid Family of mTOR Inhibitors in Salinispora Bacteria. J Am Chem Soc (2011), 133(34):13311-13313. doi: 10.1021/ja205655w.

Mincer TJ, Jensen PR, Kauffman CA, Fenical W: Widespread and persistent populations of a major new marine actinomycete taxon in ocean sediments. Appl Environ Microbiol (2002), 68(10):5005-11. doi: 10.1128/aem.68.10.5005-5011.2002.

Molinski TF, Dalisay DS, Lievens SL, Saludes JP: Drug development from marine natural products. Nat Rev Drug Discov (2009), 8(1):69:85. doi: 10.1038/nrd2487.

Momose I, Kawada M: The therapeutic potential of microbial proteasome inhibitors. Int Immunopharmacol (2016), 37:23-30. doi: 10.1016/j.intimp.2015.11.013.

Moreno-Cinos C, Goossens K, Salado IG, Van Der Veken P, De Winter H, Augustyns K: ClpP protease, a promising antimicrobial target. Int J Mol Sci (2019), 20(9). doi: 10.3390/ijms20092232.

Onaka H: Novel antibiotic screening methods to awaken silent or cryptic secondary metabolic pathways in actinomycetes. J Antibio (2017), 70(8):865-870. doi: 10.1038/ja.2017.51.

Owens RA, Hammel S, Sheridan KJ, Jones GW, Doyle S: A proteomic approach to investigating gene cluster expression and secondary metabolite functionality in Aspergillus fumigatus. PLoS One (2014), 9(9):e106942. doi: 10.1371/journal.pone.0106942. 
608

609

610

611

612

613

614

615

616

617

618

619

620

621

622

623

624

625

626

627

628

629

630

631

632

633

634

635

636

637

638

639

640

641

642

643

644

645

646

647

648

649

650

651

652

653

654

Patin NV, Duncan KR, Dorrestein PC, Jensen PR: Competitive strategies differentiate closely related species of marine actinobacteria. ISME J (2016), 10(2):478-90. doi: 10.1038/ismej.2015.128.

Patin NV, Floros DJ, Hughes CC, Dorrestein PC, Jensen PR: The role of inter-species interactions in Salinispora specialized metabolism. Microbiology (2018), 164(7):946-955. doi: 10.1099/mic.0.000679.

Patin NV, Schorn M, Aguinaldo K, Lincecum T, Moore BS, Jensen PR: Effects of Actinomycetes secondary metabolites on sediment microbial communities. Appl Environ Microbiol (2017), 83(4). doi: 10.1128/AEM.02676-16.

Penn K, Jenkins C, Nett M, Udwary DW, Gontang EA, McGlinchey Rp, Foster B, Lapidus A, Podell S, Allen EE, Moore BS, Jensen PR: Genomic islands link secondary metabolism to functional adaptation in marine Actinobacteria. ISME J (2009), 3(10):1193-203. doi: 10.1038/ismej.2009.58.

Probert I, Houdan A: The Laboratory Culture of Coccolithophores. In: Thierstein H.R., Young J.R. (eds) Coccolithophores. Springer, Berlin, Heidelberg (2004). doi: https://doi.org/10.1007/978-3-662-06278-4_9.

Reen FJ, Romano S, Dobson ADW, O'Gara F: The sound of silence: activating silent biosynthetic gene clusters in marine microorganisms. Mar Drugs (2015), 13(8):4754-4783. $10.3390 / \mathrm{md} 13084754$.

Richter TKS, Hughes CC, Moore BS: Sioxanthin, a novel glycosylated carotenoid reveals an unusual subclustered biosynthetic pathway. Environ Microbiol (2015), 17(6):2158-2171. doi: 10.1111/1462-2920.12669.

Roberts AA, Schultz AW, Kersten RD, Dorrestein PC, Moore BS: Iron acquisition in the marine actinomycete genus Salinispora is controlled by the desferrioxamine family of siderophores. FEMS Microbiol Lett (2012), 335(2):95-103. doi: 10.1111/j.15746968.2012.02641.x.

Rutledge PJ, Challis GL: Discovery of microbial natural products by activation of silent biosynthetic gene clusters. Nat Rev Microbiol (2015), 13(8):509-23. doi: 10.1038/nrmicro3496.

Schley C, Altmeyer MO, Swart R, Müller R, Huber CG: Proteome analysis of Myxococcus xanthus by off-line two-dimensional chromatographic separation using monolithic poly(styrene-divinylbenzene) columns combined with ion-trap tandem mass spectrometry. J Proteome Res (2006), 5(10):2760-8. doi: 10.1021/pr0602489.

Sher D, Thompson JW, Kashtan N, Croal L, Chisholm SW: Response of Prochlorococcus ecotypes to co-culture with diverse marine bacteria. ISME J (2011), 5(7):1125-32. doi: 10.1038/ismej.2011.1. 
Singh S, Prasad P, Subramani R, Aalbersberg W: Production and purification of a bioactive substance against multi-drug resistant human pathogens from the marine-sponge-derived Salinispora sp. Asian Pac J Trop Biomed (2014), 4(10):825-831. doi: 10.12980/APJTB.4.2014C1154.

Slattery M, Rajbhandari I, Wesson K: Competition-mediated antibiotic induction in the marine bacterium Streptomyces tenjimariensis. Microb Ecol (2001), 41(2);90-96. doi: $10.1007 / \mathrm{s} 002480000084$.

Snoberger A, Anderson RT, Smith DM: The proteasomal ATPases use a slow but highly processive strategy to unfold proteins. Front Mol Biosci (2017). 4:18. doi: 10.3389/fmolb.2017.00018.

Süssmuth RD, Mainz A: Nonribosomal peptide synthesis-principles and prospects. Angew Chem Int Ed Engl (2017), 56(14):3770-3821. doi: 10.1002/anie.201609079.

Thomy D, Culp E, Adamek M, Cheng EY, Ziemert N, Wright GD, Sass P, Brötz-Oesterhelt H: The ADEP biosynthetic gene cluster in Streptomyces hawaiiensis NRRL 15010 reveals an accessory ClpP gene as a novel antibiotic resistance factor. Appl Environ Microbiol (2019), 85(20). doi: 10.1128/AEM.01292-19.

Tuttle NR, Demko AM, Patin NV, Kapono CA, Donia MS, Dorrestein P, Jensen PR: Detection of natural products and their producers in ocean sediments. Appl Environ Microbiol (2019), 85(8). doi: 10.1128/AEM.02830-18.

Udwary DW, Zeigler L, Asolkar RN, Singan V, Lapidus A, Fenical W, Jensen PR, Moore BS: Genome sequencing reveals complex secondary metabolome in the marine actinomycete Salinispora tropica. Proc Natl Acad Sci USA (2007), 104(25);10376-81. doi: 10.1073/pnas.0700962104.

Wilson WH, Carr NG, Mann NH: The effect of phosphate status on the kinetics of cyanophage infection in the oceanic cyanobacterium Synechococcus sp. WH7803. J Phycol (1996), 32(4). doi: 10.1111/j.0022-3646.1996.00506.x.

Wilson ZE, Brimble MA: Molecules derived from the extremes of life. Nat Prod Rep (2009), 26(1):44-71. doi: 10.1039/b800164m.

Zhao P, Gu W, Huang A, Wu S, Liu C, Huan L, Gao S, Xie X, Wang G: Effect of iron on the growth of Phaeodactylum tricornutum via photosynthesis. J phycol (2018), 54(1):34-43. doi: 10.1111/jpy.12607. 
bioRxiv preprint doi: https://doi.org/10.1101/2020.05.18.103358; this version posted May 21, 2020. The copyright holder for this preprint (which was not certified by peer review) is the author/funder, who has granted bioRxiv a license to display the preprint in perpetuity. It is made available under aCC-BY 4.0 International license.

Table 1 | Biosynthetic gene clusters of Salinispora tropica CNB-440. Table shows the characterized (in orange) and orphan (in green) BGCs of S. tropica CNB-440.

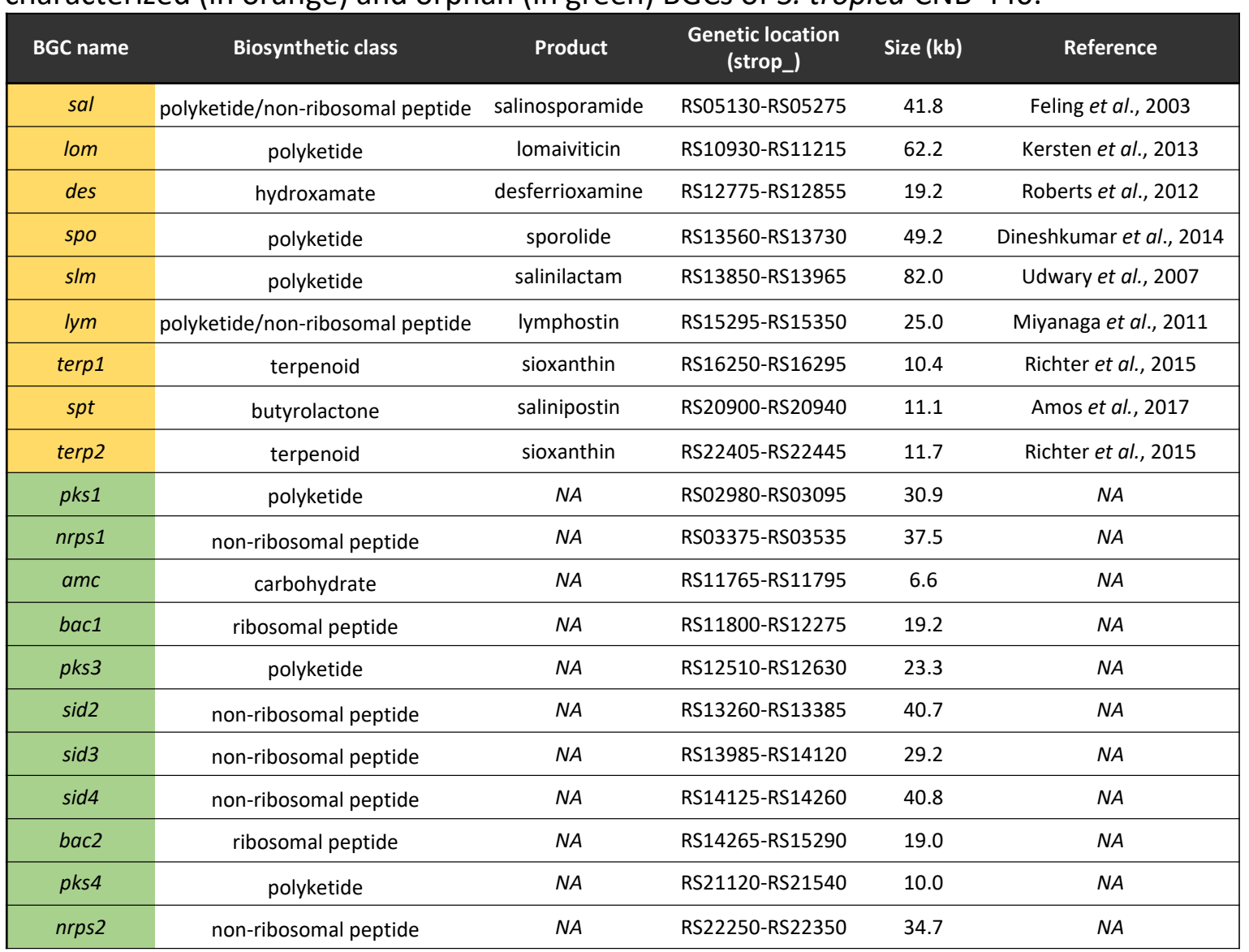


A

Culture type $=\mathrm{O}$ Axenic $\mathrm{O}=$ Co-culture
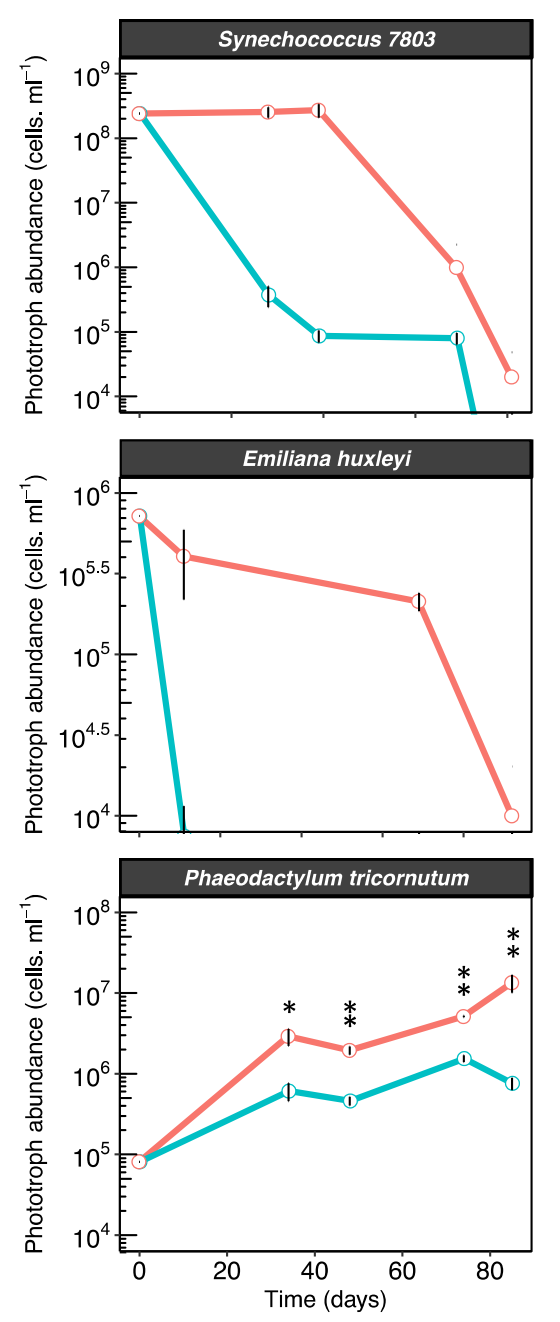

B
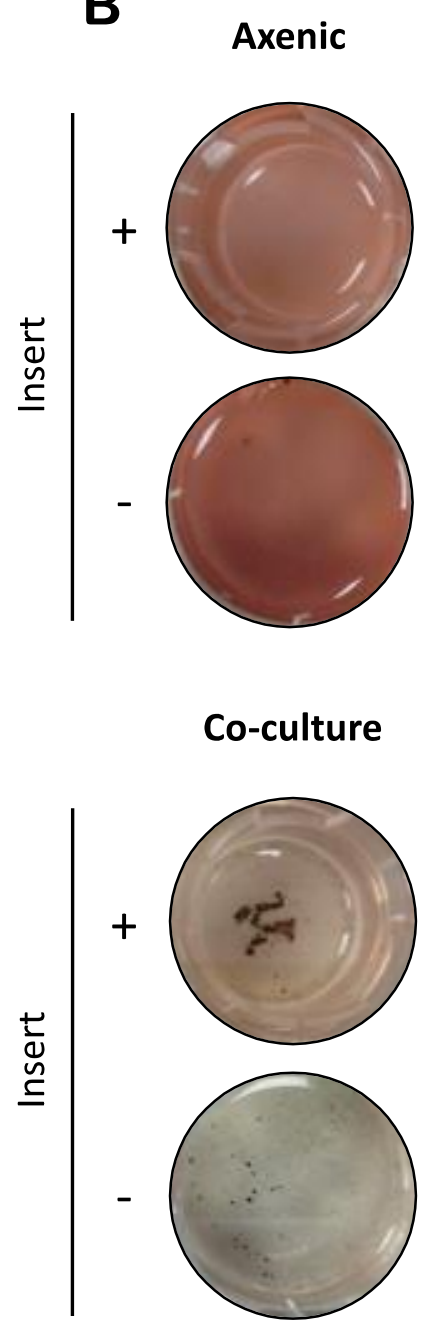

Figure 1 | Salinispora tropica inhibits the growth of marine phototrophs via the secretion of an antimicrobial molecule (A) S. tropica inhibits marine phototrophs growth in coculture. Cultures of three marine phototrophs grown axenically (red lines) and in co-culture with Salinispora tropica (blue lines). Graph shows mean \pm standard deviation of three biological replicates. Statistically significant cell abundances are indicated (T-test, significant $*$ at $p$-value $<0.05$ and ${ }^{* *}$ at $p$-value $<0.01$ ). (B) Synechococcus growth inhibition by $S$. tropica mediated by a diffusible molecule. The cyanobacterium was grown axenically and in co-culture with S. tropica, separated by a $0.4 \mu \mathrm{m}$ pore membrane insert. Photographs of representative cultures of three biological replicates are shown, 7 days after inoculation. Red pigmentation is characteristic of healthy Synechococcus cells, while cell bleaching indicates cell death. 

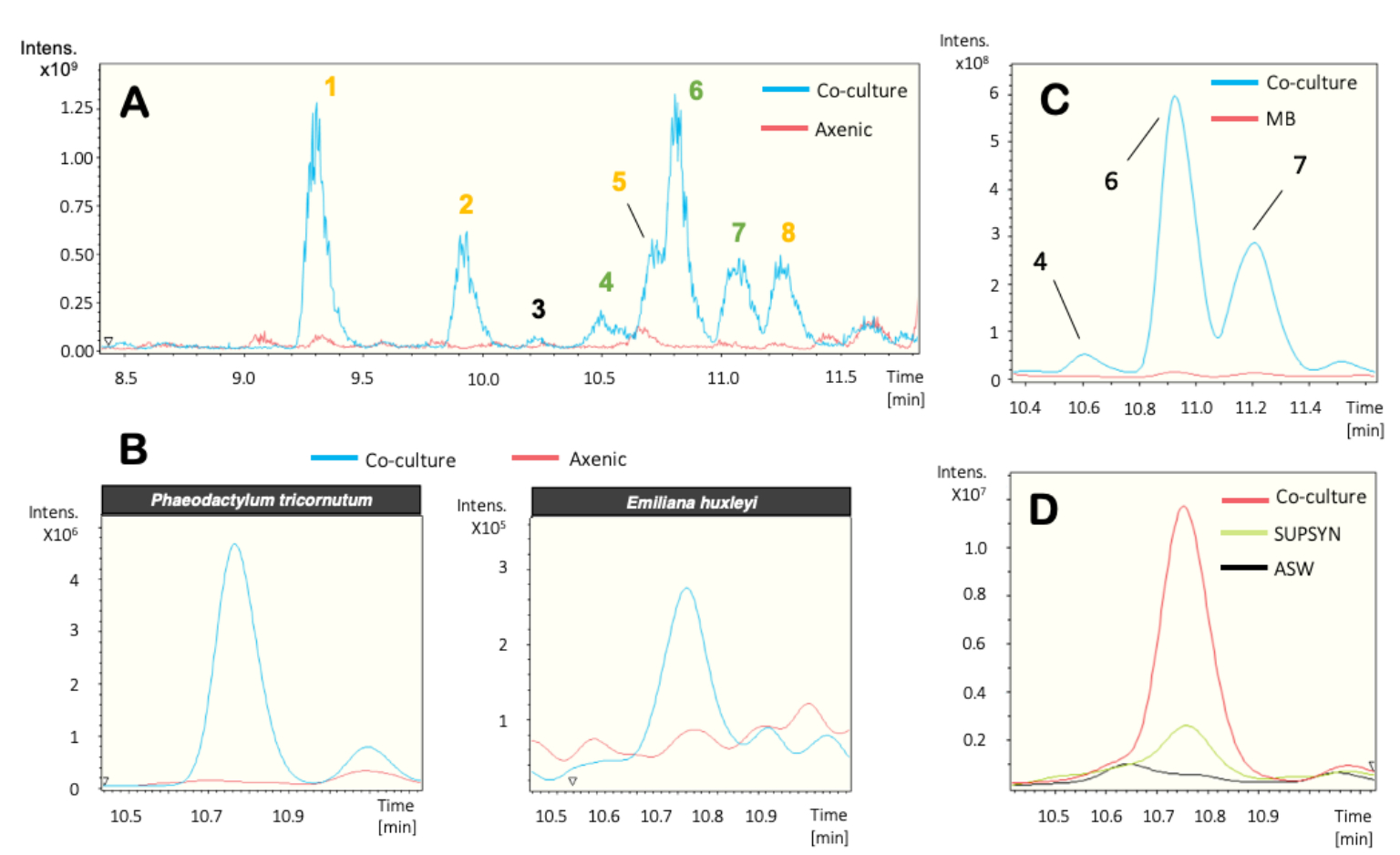

Figure 2 | Marine phototrophs trigger the production of cryptic molecules in S. tropica (A) S. tropica produces detectable small molecules in co-culture with Synechococcus. Overlaid base peak chromatograms (BPCs) of Synechococcus culture concentrated supernatants, when grown in artificial sea water (ASW) either axenically (red) or in coculture with S. tropica (blue). Peaks characteristic of the co-culture condition are labelled from 1 to 8. Color of the labels indicate groups of related compounds. (B) Other marine phototrophs also trigger the production of metabolite 6 by S. tropica as observed in panel A. Figure shows extracted ion chromatograms for the molecule 6 EIC $435.2 \pm 0.1$ in the supernatants of the phototrophs grown axenically (red) and in co-culture with $S$. tropica (blue). (C) The production of the related molecules 4, $\mathbf{6}$ and $\mathbf{7}$ is dependent on the presence of photosynthate rather than high-nutrient availability. Graph shows extracted ion chromatograms for all three cryptic molecules EIC $(464.2 ; 435.2 ; 449.2) \pm 0.5$ in the concentrated supernatants of $S$. tropica grown axenically in marine broth (MB, red) or in co-culture with Synechococcus in ASW (Co-culture, blue). (D) Cryptic molecule production is triggered by nutrients released by Synechococcus rather than cell-to-cell interactions. Graph shows extracted ion chromatograms for the cryptic molecule 6 (EIC $435.2 \pm 0.5$ ) in the supernatant of $S$. tropica grown axenically either in artificial sea water (ASW, black line) or in a conditioned Synechococcus supernatant (SUPSYN, green line); and in co-culture with Synechococcus (Co-culture, red line). 
Table 2 | Characteristics of the cryptic molecules. MS Peak numbering is based on HPLC retention time. High-resolution LC$(+)$ ESI-MS $m / z$ values and predicted chemical formulae for $[\mathrm{M}+\mathrm{H}]^{+}$ are provided.

\begin{tabular}{|c|c|c|c|}
\hline MS Peak & Observed $m / z$ & $\begin{array}{l}\text { Chemical formulae for }[\mathrm{M}+\mathrm{H}]^{+} \\
\text {(calculated } \mathrm{m} / \mathrm{z} ; \text { err }[\mathrm{ppm}] \text { ) }\end{array}$ & MS/MS \\
\hline \multirow{2}{*}{3} & \multirow{2}{*}{438.1701} & \multirow{2}{*}{$\begin{array}{c}{\left[\mathrm{C}_{28} \mathrm{H}_{24} \mathrm{~N} \mathrm{O}_{4}\right]^{+}} \\
(438.1700 ;-0.3)\end{array}$} & 194.0817 \\
\hline & & & 177.1279 \\
\hline \multirow{3}{*}{4} & \multirow{3}{*}{464.2509} & \multirow{3}{*}{$\begin{array}{l}{\left[\mathrm{C}_{22} \mathrm{H}_{34} \mathrm{~N}_{5} \mathrm{O}_{6}\right]^{+}} \\
(464.2504 ;-1.2)\end{array}$} & 276.1600 \\
\hline & & & 171.0880 \\
\hline & & & 154.0615 \\
\hline \multirow{3}{*}{6} & \multirow{3}{*}{435.2609} & \multirow{3}{*}{$\begin{array}{l}{\left[\mathrm{C}_{22} \mathrm{H}_{35} \mathrm{~N}_{4} \mathrm{O}_{5}\right]^{+}} \\
(435.2602 ;-1.7)\end{array}$} & 372.2290 \\
\hline & & & 276.1599 \\
\hline & & & 142.0979 \\
\hline 7 & 449.2764 & $\begin{array}{l}{\left[\mathrm{C}_{23} \mathrm{H}_{37} \mathrm{~N}_{4} \mathrm{O}_{5}\right]^{+}} \\
(449.2758 ;-1.3)\end{array}$ & 156.1135 \\
\hline
\end{tabular}




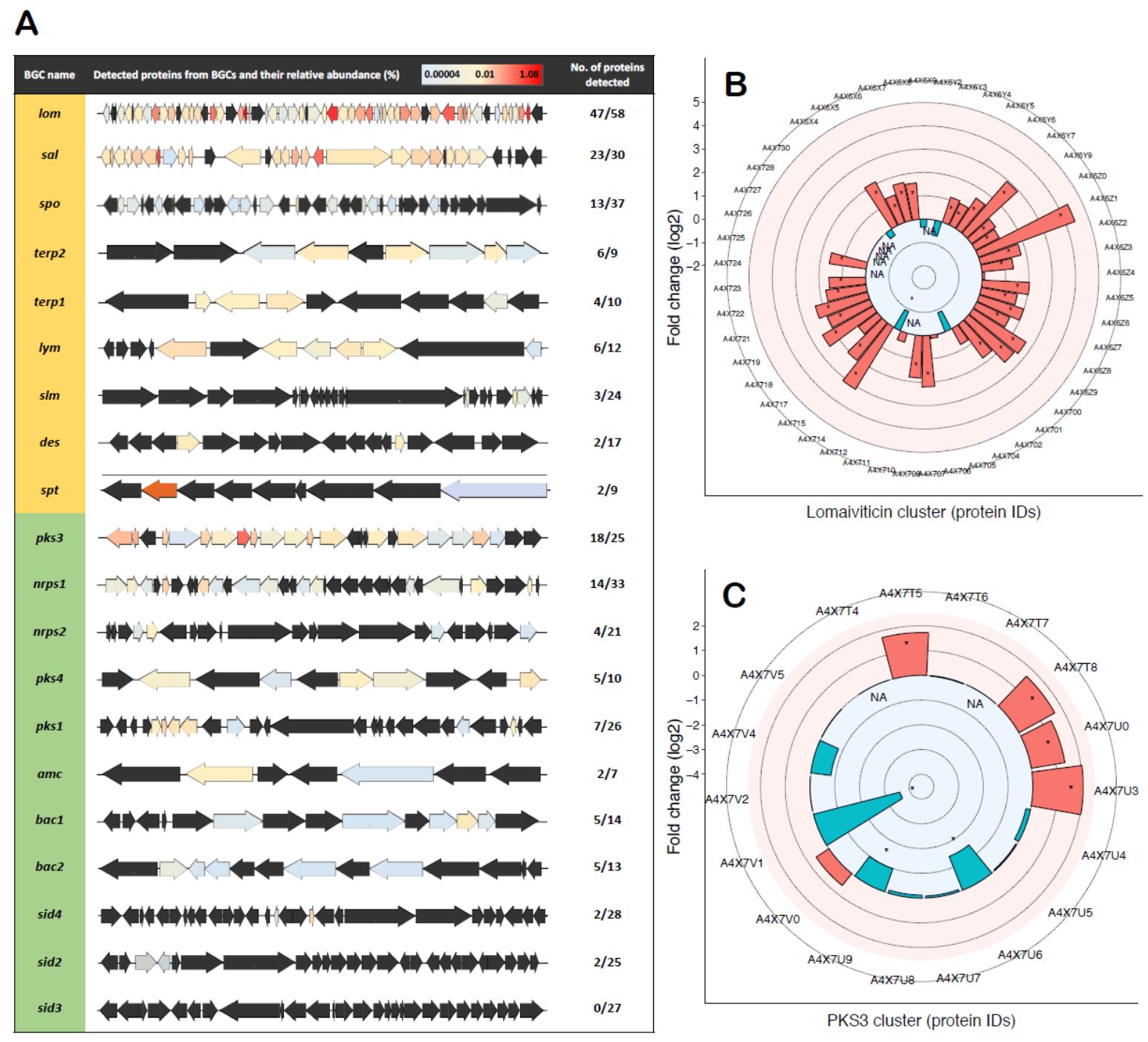

Figure 3 | Photosynthate activates orphan biosynthetic gene clusters in S. tropica. (A) Characterized (orange) and orphan BGCs (green) in S. tropica CNB-440 detected by highthroughput proteomics when grown with photosynthate (SUPSYN). Genes are colored according to the relative abundance of their corresponding proteins. Those that were not detected are represented in black. Photosynthate increased the detection of proteins involved in the production of lomaiviticin (B) and the orphan PKS3 (C) in comparison with cells grown in $\mathrm{MB}$. Up- (red) and down-regulated (blue) proteins in the presence of the photosynthate is shown. Statistically significant fold changes are indicated by an asterisk (T-test, significant at $q$-value $<0.05)$. NA indicate proteins for which the T-test and fold change could not be estimated because of missing values within a set of replicates. 
Table 3 | Detected proteins from the nrps1 orphan BGC in S. tropica CNB440 grown with photosynthate. Proteins involved in non-ribosomal peptide biosynthesis and antibiotic self-resistance are highlighted in bold.

\begin{tabular}{|lcc|}
\hline Protein ID & Annotation & $\begin{array}{c}\text { Relative abundance } \\
(\% ; n=3)\end{array}$ \\
\hline A4X2P5 & RidA family protein & 0.014 \\
\hline A4X2P8 & MFS transporter & 0.011 \\
\hline A4X2Q0 & non-ribosomal peptide synthetase & $\mathbf{0 . 0 0 4}$ \\
\hline A4X2Q2 & SDR family oxidoreductase & 0.002 \\
\hline A4X2R0 & acyl-CoA dehydrogenase & 0.005 \\
\hline A4X2R1 & acyl-CoA dehydrogenase & 0.001 \\
\hline A4X2R4 & D-alanine--poly(phosphoribitol) ligase & 0.004 \\
\hline A4X2R5 & condensation domain-containing protein & $\mathbf{0 . 0 0 1}$ \\
\hline A4X2R7 & argininosuccinate synthase & 0.001 \\
\hline A4X2R8 & methionyl-tRNA formyltransferase & 0.087 \\
\hline A4X2S2 & ATP-dependent Clp protease proteolytic subunit & $\mathbf{0 . 1 2 1}$ \\
\hline A4X2S4 & potassium channel family protein & 0.002 \\
\hline A4X2S5 & 2-oxoacid:ferredoxin oxidoreductase subunit beta & 0.006 \\
\hline A4X2S6 & 2-oxoacid:acceptor oxidoreductase subunit alpha & 0.004 \\
\hline & & \\
\hline
\end{tabular}




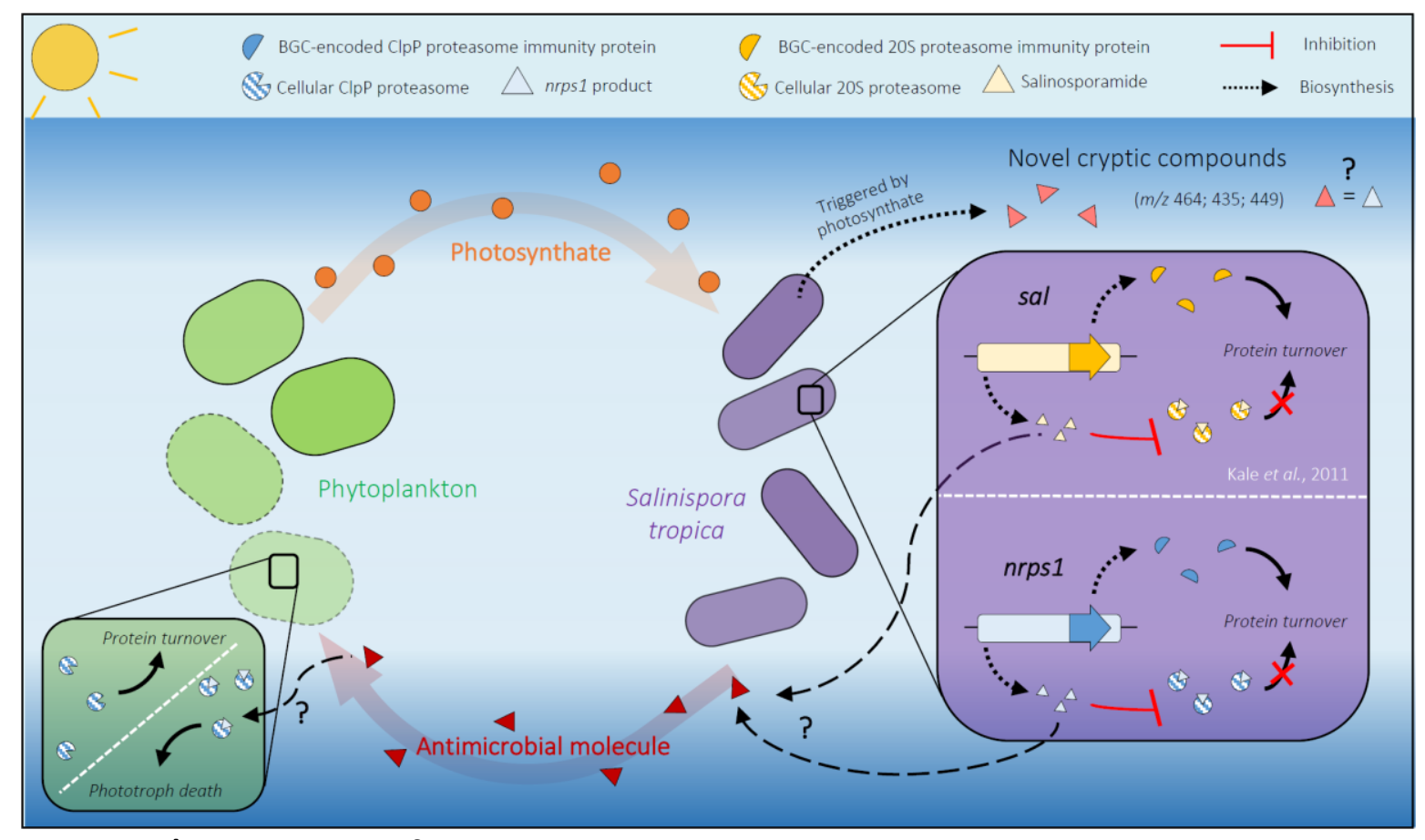

Figure 4 I Interaction of Salinispora tropica with phytoplankton. Marine phototrophs release photosynthate that triggers the biosynthesis of novel cryptic metabolites in $S$. tropica. S. tropica produces an unknown antimicrobial molecule that kills phytoplankton. The proposed mechanism of the antimicrobial metabolite as well as the activity of the nrps 1 product are depicted (green and purple boxes, respectively). The BGC nrps1 would produce a ClpP-proteasome inhibitor, to which S. tropica would be resistant because of an immunity protein encoded within the BGC, similarly to what is known for sal/salinosporamide. The nrps1-encoded proteasome inhibitor could kill the phototrophs by preventing protein turnover, leading to cell death. 